\title{
Vocal Cord Dysfunction: Analysis of 27 Cases and Updated Review of Pathophysiology \& Management
}

\author{
Shibu George ${ }^{1}$ Sandeep Suresh ${ }^{2}$ \\ ${ }^{1}$ Department of ENT, Government Medical College, Kottayam, Kerala, India \\ ${ }^{2}$ Department of ENT, Little Flower Hospital, Ernakulam, Kerala, India \\ Address for correspondence Shibu George, MS, DNB, Charivukalayil \\ (House), Ettumanoor, Kottayam 686631, Kerala, India \\ (e-mail: drshibugeorge@gmail.com; drshibugeorge@yahoo.com).
}

Int Arch Otorhinolaryngol 2019;23:125-130.

\begin{abstract}
Keywords

- Vocal Cord Dysfunction

- paradoxical vocal cord motion

- laryngopharyngeal reflux

- asthma
\end{abstract}

\section{Introduction with Objective}

Vocal cord dysfunction (VCD) is a condition characterized by paradoxical laryngeal movement resulting in inappropriate adduction of the cords, especially during the inspiration. The consequent airway obstruction results in symptoms like wheezing, stridor, dysphonia and cough, masquerading common airway diseases like asthma, vocal cord palsy and croup. ${ }^{1}$ Misdiagnosis results in unnecessary long-term medical or

received

November 29, 2016

accepted

May 6, 2018

published online

October 24, 2018
DOI https://doi.org/

10.1055/s-0038-1661358. ISSN $1809-9777$. surgical therapy and ensuing patient morbidity. Knowledge of the existence of VCD is the key to prompt diagnosis, which may be limited possibly due the relative rarity of presentation to the ENT clinic; more often, primary presentation may be to the physician or the pulmonologist. The objective of this study is to perform an evaluation of the patients diagnosed with VCD in a tertiary care ENT clinic over 5 years, and to achieve clear understanding on the pathophysiology, clinical manifestations, differential diagnoses and management of the condition.
Copyright @ 2019 by Thieme Revinter Publicações Ltda, Rio de Janeiro, Brazil
License terms

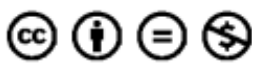




\section{Method}

A total of 27 patients who were diagnosed with VCD in the department of ENT, from January 2013 to December 2017 were included in this study after obtaining clearance from the Scientific Review Board and the Ethical Committee of the institution. A signed informed consent about the study was obtained from every patient (or from the parent, if patient was younger than 18 years of age) prior to their inclusion in this study. The patients either reported directly or were referred by physicians or pulmonologists to attend the ENT outpatient department/casualty department. They were subjected to detailed history and thorough evaluation including transnasal fiberoptic laryngoscopy examination. Patients whose symptoms were found to be due to other conditions, such as bronchial asthma, vocal cord palsy and croup, were excluded. A pulmonary function study with spirometry evaluation was performed in all patients in consultation with a pulmonologist. The findings of history and evaluation were analyzed to make a diagnosis of VCD. After initiating the treatment, the patients were kept on regular weekly follow-up for 1 month and then monthly follow-up. Assessment of symptomatic relief along with repeat laryngoscopy and spirometry were done on monthly follow ups. Minimum follow up period was 6 months. Data collected was analyzed based on distribution by demographic characteristics, presentation, associations, management and follow-up.

\section{Results}

A total of only 27 cases were diagnosed to have VCD during the past 5 years, which points to the rarity of presentation to the ENT clinic; interestingly, 14 of them were referred for opinion by general physicians or pulmonologists. A strong female preponderance was noted in the study population (19 females \& 8 males). The youngest patient in the group was a 15 -year-old male and the oldest, a 42-year-old female; the mean age was 31 (-Table 1 ).

12 patients (44\%) in this study presented with noisy breathing and stridor aggravated by stress or exertion; of these, 4 patients had severe stridor and acute airway obstruction with clinical findings masquerading bilateral vocal cord palsy. 11 patients (41\%) were being treated for refractory asthma and 3 patients (11\%) for chronic cough with intermittent wheeze by physicians or pulmonologists and referred for ENT opinion following treatment failure. Dysphonia and throat congestion were reported in $3 \%$ of the

Table 1 Vocal cord dysfunction-age \& sex distribution

\begin{tabular}{|l|l|l|}
\hline Age group (in years) & No. of males & No. of females \\
\hline $11-20$ & 1 & 2 \\
\hline $21-30$ & 3 & 6 \\
\hline $31-40$ & 4 & 10 \\
\hline $41-50$ & 0 & 1 \\
\hline Total & 8 & 19 \\
\hline
\end{tabular}

cases. The majority of the patients (66\%) had associated globus sensation and throat clearing, which is suggestive of laryngopharyngeal reflux disease (LPRD), and classical symptoms of gastroesophageal reflux, like heartburn, were reported by $19 \%$ of them. As many as $48 \%$ of the patients had strong anxiety overlay; $11 \%$ had frank depressive illness and had already sought medical advice for the same but were not on sustained medications. Associated neurological disease was noted in 1 patient (3\%); she had multiple system atrophy and developed acute airway obstruction following VCD.

Transnasal fiberoptic laryngoscopy could demonstrate paradoxical inspiratory vocal cord adduction in all patients during the attack of VCD. The demonstrable adduction was confined to the anterior two thirds of the vocal cords, with a posterior diamond-shaped glottic chink. Adduction of the false cord was also noted in 11 patients. The laryngoscopies in between the attacks were normal; however, even in this phase, the typical vocal cord changes could be induced in patients by maneuvers like hyperventilation, or a forced expiratory effort followed by rapid inspiration.

The results of the laryngoscopy could also suggest strong association with laryngopharyngeal reflux disease (LPRD), with demonstrable findings in the majority of the patients, the most common of which were posterior laryngitis (63\%) and pharyngeal wall cobble stoning (63\%). Less frequently, interarytenoid pachydermia (33\%), postnasal discharge (19\%), vocal cord edema (11\%) and pseudo sulcus (3\%) were also seen.

Pulmonary function tests (PFTs) by spirometry were performed in all patients, in consultation with a pulmonologist. A decrease in the maximal inspiratory flow rate was demonstrable in all patients during an attack, while the maximal expiratory flow rate did not change. Flow-volume loops (FVLs) demonstrated inspiratory loop truncation or flattening (indicating decrease in inspiratory flow) during symptomatic periods, suggestive of variable extra-thoracic obstruction; the expiratory loops were normal. Spirometry was normal in between attacks. (distribution of symptomatology and findings on evaluation has been presented in - Table $\mathbf{2}$ ).

Preliminary management during an acute attack of VCD included reassuring patients regarding the benign nature of the disease and creating distractions to shift their attention away from breathlessness. The patients were instructed to take rapid and shallow breaths (panting), which brought about significant reduction of the symptoms in the majority of them. Thirteen patients with clear anxiety overlay were treated with added benzodiazepines during the acute attack and responded readily. Midazolam was titrated in incremental doses to avoid oversedation and respiratory depression. Four patients who presented with acute airway obstruction and severe stridor needed added continuous positive airway pressure (CPAP) to terminate the attack.

In the long term, all 19 patients diagnosed to have associated LPRD were treated with dietary advices, lifestyle modifications and proton pump inhibitors; omeprazole, $20 \mathrm{mg}$ bis in die (BID), was prescribed for a minimum of 3 months and then put on maintenance of $20 \mathrm{mg}$ once a day (OD) for 3 additional months; there was prompt response in 15 patients who complied with the schedule. Relapse of VCD 
Table 2 Distribution of symptoms \& findings on evaluation of patients with vocal cord dysfunction.

\begin{tabular}{|c|c|c|}
\hline Evaluation & Findings & No. of Patients \\
\hline \multirow[t]{4}{*}{ History-Presentation } & a. Inspiratory/biphasic stridor (aggravated by stress, exertion, irritants) & 12 \\
\hline & b. Refractory wheezing & 11 \\
\hline & c. Chronic cough \& intermittent breathlessness & 3 \\
\hline & d. Dysphonia \& throat congestion & 1 \\
\hline \multirow[t]{5}{*}{ History-Associations } & a. Globus sensation \& throat clearing & 19 \\
\hline & b. Heartburn \& regurgitation & 5 \\
\hline & c. Anxiety \& stress & 13 \\
\hline & d. Depressive illness & 3 \\
\hline & e. Neurological disease & 1 \\
\hline \multirow{8}{*}{$\begin{array}{l}\text { Flexible transnasal } \\
\text { laryngoscopy }\end{array}$} & a. Inspiratory vocal cord adduction & 27 \\
\hline & b. False cord adduction & 11 \\
\hline & c. Posterior laryngitis & 17 \\
\hline & d. Cobble stoning pharyngeal wall & 17 \\
\hline & e. Interarytenoid pachydermia & 9 \\
\hline & f. Postnasal discharge & 5 \\
\hline & g. Vocal cord edema & 3 \\
\hline & h. Pseudosulcus & 1 \\
\hline Spirometry & $\begin{array}{l}\text { During attack } \\
\text { Variable extra thoracic obstruction } \\
\text { (truncated/flattened inspiratory flow-volume loops) } \\
\text { Between attacks-Normal }\end{array}$ & 27 \\
\hline
\end{tabular}

was noted in four patients, primarily following premature discontinuation of medications, but improved upon restarting therapy. The treatment response was judged based on resolution of the symptoms and normal follow-up fiberoptic laryngoscopy and spirometry findings.

All patients with anxiety overlay were sent for speech therapy, psychiatric evaluation and counseling. Antidepressants and antianxiety medications were started in three patients diagnosed with frank depressive illness and two patients with posttraumatic stress disorder, while others responded readily to speech therapy and counseling alone.

\section{Discussion}

Along with protection of lower airways and phonation, respiration is a vital function of the larynx. The act of increasing and decreasing the diameters of the larynx has an active influence on inspiration, expiration and resulting lung volume. During normal inspiration, the vocal cords abduct to allow air into the lung due to action of posterior cricoarytenoid muscle controlled by the medullary respiratory center through Vagus. Likewise, there is a partial adduction of the cords during expiration due to relaxation of the posterior cricoarytenoid coupled with the contraction of the lateral cricoarytenoid muscle; this allows outward movement of air and maintenance of alveolar patency by providing positive end expiratory pressure. Vocal cord dysfunction, also known as paradoxical vocal cord motion, is characterized by inappropriate vocal cord adduction during the respiratory cycle (especially inspiration) leading to intermittent episodes of acute functional airway obstruction. Its symptoms closely mimic wheezing or stridor and the condition is frequently misdiagnosed as asthma or upper airway obstruction. It can so much mimic asthma that it is also called 'vocal cord asthma'. The $1^{\text {st }}$ modern case report of VCD evidenced by laryngoscopy was made in 1974 by Patterson et al, ' for which he coined the term 'Munchausen's stridor'. The exact mechanism that causes glottis chink narrowing or intermittent inspiratory closure, independent of any changes in the lower airway caliber, is unknown. The larynx receives extensive sensory and motor innervation which may become hyperexcitable and hyperresponsive due to repeated stimulation by intrinsic or extrinsic factors; this lowers the threshold for reflex closure of the vocal cords. A myriad range of factors has been postulated, which include irritant exposure, occupational pollutants, psychological stress, malingering and neurological causes.

This condition has been predominantly observed in females; in our study, the incidence among female patients was more than twice the incidence in males. Systematic literature reviews on VCD have identified similar male to female ratio; 1:3 (Brugman ${ }^{2}$ ) and 1:2 (Morris et $\mathrm{al}^{3}$ ). The mean age of the patients in our study was 31 , with $50 \%$ of the patients between 30 and 40 years. The typical age group of VCD cited in the literature is 20 to 40 years, but the condition 
has been reported in age groups ranging between the $1^{\text {st }}$ and $8^{\text {th }}$ decade of life. Brugman's ${ }^{2}$ systematic review has reported increased incidence of VCD among children and adolescents; $35 \%$ of the patients were children with a median age of 14 . In our study, however, only $14 \%$ of the patients were younger than 20 years of age, with the youngest being 15 years old.

Irritant induced VCD was described by Perkner et $\mathrm{al}^{4}$; environmental or occupational irritants, such as ammonia, cleaning chemicals, organic solvents, flux flames or smoke, were implicated. Alteration of vagal mediated laryngeal tone has been hypothesized to cause the lowering of the threshold for irritant stimuli producing inspiratory adduction. However, the major irritants to cause an irritable larynx and paradoxical vocal cord movement have been identified to be the laryngopharyngeal reflux disease (LPRD) and the upper aerodigestive tract manifestations of gastroesophageal reflux disease (GERD). The mechanism involved may be accentuation of the glottis closure reflux secondary to acid damage of the laryngeal mucosa. Patients with VCD were found to have patches of heterotrophic gastric mucosa in the lower esophagus along with posterior laryngitis. The majority of the patients with VCD, in a series by Powell et al, ${ }^{5}$ had laryngopharyngeal manifestations of reflux. Murry et $\mathrm{al}^{6}$ described five cases of VCD in which the patients presented with refractory cough due to LPRD. In our study population, $66 \%$ of the patients had symptoms of LPRD, mainly globus sensation and throat clearing. Classical reflux symptoms, such as heartburn and regurgitation, were seen only in $19 \%$ of the patients. This implies that LPRD is a more important entity than GERD in triggering VCD. Significantly, laryngopharyngeal changes could be demonstrable in all of these patients on fiberoptic laryngoscopy, with the most common being posterior laryngitis and cobble-stoning of the pharyngeal wall (-Table 2). In comparison with the esophagus, the laryngeal mucosa is more sensitive to the refluxate, since it is not protected by peristalsis or buffered by salivary bicarbonate. Morrison et $\mathrm{al}^{7}$ had described 'irritable laryngeal syndrome' following acid exposure of the larynx due to reflux, causing paradoxical adduction of the cords. The absence of clear gold standard diagnostic criteria for LPRD in the literature probably hinders postulation of a direct causal relation with VCD.

According to Leo and Konakanchi, ${ }^{8}$ stress, emotions, and psychiatric disturbances, like hysterical conversion or anxiety disorders, contribute to VCD. The literature search reveals high incidence of VCD in psychiatric conditions. Lacy and McManis ${ }^{9}$ reviewed 45 cases of VCD in which psychiatric disorders were reported; $52 \%$ had a conversion disorder, $13 \%$ had major depression and $10 \%$ had a factitious disorder. Underlying all these diagnoses was the presence of significant emotional stress. In our study, there were three patients who were already diagnosed to have depressive illness and treated for the same. More significantly, anxiety and underlying stress could be clearly elicited by history in 13 patients; of these, 2 patients with posttraumatic stress needed drug treatment. Anxiety attacks may be integral to patients with other airway diseases and are found to precede the respiratory symptoms; in contrast, it persisted along with respiratory symptoms in VCD. Although the majority of these cases are purely subconscious, it should be kept in mind that voluntarily inducing vocal cord adduction with an intention of secondary gain is not uncommon among malingerers.

Neurological causes, though uncommon, should be ruled out in all cases of VCD. Van der Graaff et $\mathrm{al}^{10}$ reported that neurological diseases, like amyotrophic lateral sclerosis, causes VCD, while Maschka et $\mathrm{al}^{11}$ described brainstem compression or cortical injury to result in VCD. Other diseases implicated include cerebrovascular accidents, ArnoldChiari malformation and myasthenia gravis. One patient in our study had multi system neurological atrophy presenting with VCD and acute onset stridor.

Due to the remarkable similarity of presentation, VCD is very frequently diagnosed as asthma and probably amounts to $\sim 10 \%$ of the patients at specialized centers seeking evaluation for resistant asthma. Among the 49 cases of vocal cord dysfunction described by Doshi and Weinberger, ${ }^{12} 41$ cases were incorrectly treated for asthma. Newman et $\mathrm{al}^{13}$ conducted a retrospective analysis of 95 patients with intractable asthma and found 10\% had VCD and 30\% had VCD coexisting with asthma. These patients were treated with inhaled corticosteroids for an average time period of 4.8 years before receiving the diagnosis of VCD. An otolaryngology opinion is hence prudent in every patient with wheezing once the diagnosis of asthma has been ruled out. In our series, 11 patients with refractory asthma unresponsive to treatment and 3 patients with intermittent wheezing were referred by pulmonologists when spirometry demonstrated extrathoracic obstruction.

Vocal cord dysfunction may also present directly to the otolaryngologist as stridor masquerading other organic causes of upper airway obstruction which includes unilateral or bilateral vocal cord palsy, laryngospasm, laryngomalacia, acute epiglottitis, croup, foreign body obstruction, angioedema or anaphylaxis. Appropriate treatment is possible only if accurate diagnosis is made during an attack. Procedures for emergency airway including intubation or even tracheostomy have been initiated on patients with VCD following misdiagnosis. In the study by Newman et al, ${ }^{13}$ as high as $28 \%$ patients with VCD were mismanaged and were intubated. In our series 12 patients presented with stridor; 4 among them had severe symptoms that at one stage made us contemplate endotracheal intubation.

The trigger of paradoxical vocal cord movement and stridor varies. Stress and anxiety, particularly in social situations, are clearly triggers in the majority of the patients. Powell et $\mathrm{al}^{5}$ described 22 cases of juvenile VCD in which social stress due to involvement in sports activities was observed in 12 patients. Exercise may be the predominant trigger in some. Vocal cord dysfunction may present as exercise-induced shortness of breath or stridor that mimic bronchospasm; the amount of exercise to induce VCD may vary per individual. Exercise-induced VCD was diagnosed in 19 out of the 370 athletes screened by Rundell et al. ${ }^{14}$ Protracted reflux especially aggravated by dietary habits is another trigger and so is inhalation of perfumes and strong 
odors. In our series, such a trigger could be observed in all the 10 patients presenting as stridor; the most common trigger observed was stress ( 6 patients) followed by physical exertion (3 patients). One patient, interestingly, reported incense fume inhalation during religious rituals as her trigger for developing breathlessness and noisy breathing. Because of the inspiratory adduction, the speech pattern is usually broken and may be the presentation at times; we had one patient who presented with dysphonia and throat congestion.

The initial otolaryngology evaluation may reveal inspiratory or biphasic stridor with strained voice which may immediately suggest an organic laryngeal lesion to the unsuspecting clinician. The classical finding on laryngoscopy is adduction of the anterior two thirds of the vocal cord, with a posterior chinking or diamond shaped margination between the posterior part of the cords during inspiration, early phases of expiration or both. It goes without saying that the impression conveyed upon initial evaluation is of a critical glottic space. Adduction occurring during the end expiration, on the other hand, is purely physiological and may be visualized in normal individuals and should not be confused with VCD. Furthermore, during laryngoscopy, especially upon mirror examination, a protracted gag reflux or cough can cause adduction of the cords and hence, it should be taken into consideration on arriving at a diagnosis. Thus, the investigation of choice would be a transnasal flexible fiberoptic laryngoscopy in which the diagnosis can be clinched on persistent viewing of the cords during all phases of respiration. Inspiratory vocal cord adduction with posterior glottic chink was demonstrable in all patients in our study during the attack; vocal cord movements were normal in between the attacks. As discussed earlier, laryngoscopy also provided objective evidence of LPRD in these patients, as well.

Pulmonary function test and spirometry during the attack reveal a pattern of variable extrathoracic airway obstruction with truncated or flattened inspiratory FVL. Morris ${ }^{15}$ et al reviewed 1,500 cases of VCD in the published literature and found that $28 \%$ had FVL truncation on spirometry. However, the predictive value of the FVL to diagnose VCD has not been evaluated prospectively. Symptomatic VCD may result in reduction in both the forced expiratory volume $\left(\mathrm{FEV}_{1}\right)$ and forced vital capacity (FVC); however, the $\mathrm{FEV}_{1} / \mathrm{FVC}$ ratio is normal. Spirometry is normal in between attacks. Chest radiographs are generally not helpful in establishing a diagnosis of VCD.

Emergency management: Reybet ${ }^{16}$ proposed use of a continuous positive airway pressure(CPAP) device delivering intermittent pulses to resolve an acute attack, since it can widen the glottis and act as a pneumatic splint to reduce turbulence. Four patients in our series who needed emergency management for severe airway obstruction were treated with CPAP and readily improved. Heliox therapy has been recommended by Reisner and Borish, ${ }^{17}$ as early as 1995 , for immediate relief of the respiratory distress in VCD. Being an 80:20 mixture of helium and oxygen, it is less dense than air and on inhalation, it reduces airway turbulence to bring down respiratory effort while eliminating respiratory noise, which allays anxiety. Benzodiazepines and reassurance reduces anxiety and thus may be useful, especially in the prevention of propagation of the attack. Topical lidocaine, if applied to the larynx, breaks the hyperactive glottal and supraglottal muscle contractions and opens the larynx. Superior laryngeal nerve block has been tried in more severe cases; In such cases, an intra laryngeal injection of Botulinum toxin has been proposed as an option, since it causes neuromuscular blockade and laryngeal muscle weakness to facilitate airflow as advocated by Maillard et $\mathrm{al}^{18}$ and Altman et al. ${ }^{19}$

In cases of long-term therapy, the cause of the paroxysm should be elicited. A significant number of cases are either due to LPRD or is associated with GERD, and may need aggressive treatment with proton pump inhibitors and appropriate life style modifications. All patients with LPRD in our series responded well to treatment continued for minimum of 6 months. There was a relapse of VCD in four patients who discontinued treatment for LPRD but improved on restarting therapy. According to Murry et $\mathrm{al}^{6}{ }^{6}$ the symptoms resolved in an average time period of 8.2 months after treatment with proton pump inhibitors and respiratory retraining therapy. A history of prior exposure to occupational or environmental irritants should be obtained and avoidance is advised against the same. VCD secondary to neurological disease should be managed along with treatment of the predisposing condition. Kent and Balkissoon ${ }^{20}$ reaffirm that a multidisciplinary approach should be adopted in the general management, which includes speech therapy, psychotherapy and patient education. Speech therapy techniques are aimed at expiration and abdominal breathing than inspiration and laryngeal breathing with pertinent advices on relaxed throat breathing pattern. In a study done by Sullivan et $\mathrm{al}^{21}$ in 20 female athletes, symptoms of VCD during exercise could be controlled in $95 \%$ of the study subjects by means of speech therapy for up to 6 months following treatment. Mathers et $\mathrm{al}^{22}$ described inspiratory muscle training (IMT) resulting in increased inspiratory muscle strength, thus improving effort tolerance as a treatment modality for exercise-induced VCD. Panting as a maneuver can lead to physiological increase in the glottic aperture by relaxation of the muscles and laryngeal adduction. According to a systematic review of psychological interventions for VCD by Guglani et al, ${ }^{23}$ techniques such as psychotherapy, behavioral therapy, antianxiety and antidepressant medications, and hypnotherapy in conjunction with breathing exercises are effective options. These allow patients to search for the potential causes and use relaxation techniques to combat anxiety and stress.

Therefore, the initial response to standard treatment is good in VCD, provided that the diagnosis is made early in the evolution of the symptoms. Misdiagnosis is common and often results in overtreatment, which unfortunately may cause escalated health care costs and even long-term morbidity for the patient.

\section{Conclusion}

Vocal cord dysfunction is often underappreciated, and, from the otolaryngologist's perspective, the most important factor in the diagnosis awareness of the condition. Vocal cord dysfunction 
can be difficult to treat, as the condition is often misdiagnosed in clinical practice. The primary presentation as stridor may often be dramatic, mimicking an airway emergency. More commonly, it mimics asthma and presents to a general physician or pulmonologist followed by secondary referrals. Fiberoptic laryngoscopy is the diagnostic gold standard to demonstrate paradoxical adduction of vocal cords during an attack. It is prudent to consider VCD in those patients initially regarded as 'asthmatics' but who are now suspected to have 'stridor,' since the actual incidence of VCD should be much more than the diagnosed cases. The role of acid reflux as the most fashionable villain in the upper airway is not an overstatement, since LPRD plays a definite role in the pathogenesis of VCD. Other intrinsic and extrinsic triggers can exacerbate laryngeal hyperresponsiveness and focusing on minimizing such triggers can also serve to improve symptoms. A multidisciplinary approach should be adopted for the management, which should be specific for each individual patient.

\section{Funding}

There was no funding for this study.

Conflicts of Interest

The authors declare that they have no conflicts of interest.

\section{References}

1 Patterson R, Schatz M, Horton M. Munchausen's stridor: nonorganic laryngeal obstruction. Clin Allergy 1974;4(03):307-310

2 Brugman S. The many faces of vocal cord dysfunction. What 36 years of literature tells us. Am J Respir Crit Care Med 2003;167:A588

3 Morris MJ, Allan PF, Perkins PJ. Vocal cord dysfunction: aetiologies and treatment. Clin Pulm Med 2006;13:73-86

4 Perkner JJ, Fennelly KP, Balkissoon R, et al. Irritant-associated vocal cord dysfunction. J Occup Environ Med 1998;40(02):136-143

5 Powell DM, Karanfilov BI, Beechler KB, Treole K, Trudeau MD, Forrest LA. Paradoxical vocal cord dysfunction in juveniles. Arch Otolaryngol Head Neck Surg 2000;126(01):29-34

6 Murry T, Tabaee A, Aviv JE. Respiratory retraining of refractory cough and laryngopharyngeal reflux in patients with paradoxical vocal fold movement disorder. Laryngoscope 2004;114(08): 1341-1345

7 Morrison M, Rammage L, Emami AJ. The irritable larynx syndrome. J Voice 1999;13(03):447-455

8 Leo RJ, Konakanchi R. Psychogenic Respiratory Distress: A Case of Paradoxical Vocal Cord Dysfunction and Literature Review. Prim Care Companion J Clin Psychiatry 1999;1(02):39-46

9 Lacy TJ, McManis SE. Psychogenic stridor. Gen Hosp Psychiatry 1994;16(03):213-223

10 van der Graaff MM, Grolman W, Westermann EJ, et al. Vocal cord dysfunction in amyotrophic lateral sclerosis: four cases and a review of the literature. Arch Neurol 2009;66(11):1329-1333

11 Maschka DA, Bauman NM, McCray PB Jr, Hoffman HT, Karnell MP, Smith RJ. A classification scheme for paradoxical vocal cord motion. Laryngoscope 1997;107(11 Pt 1):1429-1435

12 Doshi DR, Weinberger MM. Long-term outcome of vocal cord dysfunction. Ann Allergy Asthma Immunol 2006;96(06):794-799

13 Newman KB, Dubester SN. Vocal cord dysfunction: masquerader of asthma. Semin Respir Crit Care Med 1994;15:161-167

14 Rundell KW, Spiering BA. Inspiratory stridor in elite athletes. Chest 2003;123(02):468-474

15 Morris MJ, Allan PF, Perkins PJ. Vocal cord dysfunction: etiologies and treatment. Clin Pulm Med 2006;13(02):73-86

16 Reybet-Degat O. [Pathology of craniocervical junction and sleep disorders]. Rev Neurol (Paris) 2001;157(11 Pt 2):S156-S160

17 Reisner C, Borish L. Heliox therapy for acute vocal cord dysfunction. Chest 1995;108(05):1477

18 Maillard I, Schweizer V, Broccard A, Duscher A, Liaudet L, Schaller MD. Use of botulinum toxin type A to avoid tracheal intubation or tracheostomy in severe paradoxical vocal cord movement. Chest 2000;118(03):874-877

19 Altman KW, Mirza N, Ruiz C, Sataloff RT. Paradoxical vocal fold motion: presentation and treatment options. J Voice 2000;14(01): 99-103

20 Kenn K, Balkissoon R. Vocal cord dysfunction: what do we know? Eur Respir J 2011;37(01):194-200

21 Sullivan MD, Heywood BM, Beukelman DR. A treatment for vocal cord dysfunction in female athletes: an outcome study. Laryngoscope 2001;111(10):1751-1755

22 Mathers-Schmidt BA, Brilla LR. Inspiratory muscle training in exercise-induced paradoxical vocal fold motion. J Voice 2005;19 (04):635-644

23 Guglani L, Atkinson S, Hosanagar A, Guglani L. A systematic review of psychological interventions for adult and pediatric patients with vocal cord dysfunction. Front Pediatr 2014;2:82 